\title{
ESO Large Program on physical studies of Trans-Neptunian objects and Centaurs: Final results of the visible spectrophotometric observations ${ }^{\star}$
}

\author{
S. Fornasier ${ }^{1}$, A. Doressoundiram ${ }^{2}$, G. P. Tozzi ${ }^{3}$, M. A. Barucci ${ }^{2}$, H. Boehnhardt ${ }^{4}$, \\ C. de Bergh ${ }^{2}$, A. Delsanti ${ }^{2}$, J. Davies ${ }^{5}$, and E. Dotto ${ }^{6}$ \\ 1 Astronomy Department of Padova University, Vicolo dell'Osservatorio 2, 35122 Padova, Italy \\ 2 LESIA, Observatoire de Paris, 92195 Meudon Pricipal Cedex, France \\ e-mail: [alain.doressoundiram; antonella.barucci; Catherine.deBergh;Audrey.Delsanti]@obspm.fr \\ 3 INAF-Osservatorio Astrofisico di Arcetri, Largo E. Fermi 5, 50125 Firenze, Italy \\ e-mail: tozzi@arcetri.astro.it \\ ${ }^{4}$ Max-Planck-Institute for Astronomy, Koenigstuhl 17, 69117 Heidelberg, Germany \\ e-mail: hboehnha@mpia-hd.mpg.de \\ 5 Astronomy Technology Centre, Blackford Hill, Edinburgh, EH9 3HJ, Scotland, UK \\ e-mail: J.Davies@roe.ac.uk \\ ${ }^{6}$ INAF-Osservatorio Astronomico di Roma, via Frascati 33, 00040, Monte Porzio Catone (Roma), Italy \\ e-mail: dotto@mporzio.astro.it
}

Received 12 February 2004 / Accepted 8 April 2004

\begin{abstract}
The Large Program on physical studies of TNOs and Centaurs, started at ESO Cerro Paranal on April 2001, has recently been concluded. This project was devoted to the investigation of the surface properties of these icy bodies through photometric and spectroscopic observations. In this paper we present the latest results on these pristine bodies obtained from the spectrophotometric investigation in the visible range.

The newly obtained spectrophotometric data on 3 Centaurs and 5 TNOs, coming from 2 observing runs at the Very Large Telescope (VLT), show a large variety of spectral characteristics, comprising both gray and red objects in the two different populations. A very broad and weak absorption feature, centered around $7000 \AA$, has been revealed in the spectrum of the gray TNO 2003 AZ84. This absorption is very similar to a feature observed on low albedo main belt asteroids and attributed to the action of the aqueous alteration process on minerals. This process was previously also claimed as the most plausible explanation for some peculiar visible absorption bands observed on 2000 EB173 and 2000 GN171 in the framework of the Large Program (Lazzarin et al. 2003; de Bergh et al. 2004). This detection seems to reinforce the hypothesis that aqueous alteration might have taken place also at such large heliocentric distances.

We also report the results of a spectroscopic investigation performed outside the Large Program on the very interesting TNO 2000 GN171 during part of its rotational period. This object, previously observed twice in the framework of the Large Program, had shown during the early observations a very peculiar absorption band tentatively attributed to aqueous alteration processes. As this feature was not confirmed in a successive spectrum, we recently repeated the investigations of 2000 GN171, finding out that it has an heterogeneous composition.

Finally an analysis of the visible spectral slopes is reported for all the data coming from the Large Program and those available in literature.
\end{abstract}

Key words. Kuiper belt - minor planets, asteroids

\section{Introduction}

Trans-Neptunian objects (TNOs) are also known as Edgeworth-Kuiper objects (EKOs) in honour of the first

Send offprint requests to: S. Fornasier,

e-mail: fornasier@pd.astro.it

* Based on observations obtained at the VLT Observatory Cerro Paranal of European Southern Observatory, ESO, Chile, in the framework of programs 167.C-0340(G), 071.C-0500. scientists who hypothesed the existence of icy bodies orbiting beyond Neptune. These small bodies are presumed to be the most primitive and thermally unprocessed materials known in our planetary system and can provide clues to understand what happened in the outer solar system during the protoplanetary nebula phase.

Objects scattered out of the Edgeworth-Kuiper belt are known as Centaurs or Scattered Disk Objects. Both have highly eccentric orbits. Centaurs have unstable orbits between those 
Table 1. Observational circumstances: dynamical class (1998 SN165 does not belong to a particular class and is generally indicated as TNO), heliocentric distance $(r)$, geocentric distance $(\Delta)$ and phase angle $(\alpha)$.

\begin{tabular}{lrlccc}
\hline \hline Object & Night & Class & $\begin{array}{c}r \\
(\mathrm{AU})\end{array}$ & $\begin{array}{c}\Delta \\
(\mathrm{AU})\end{array}$ & $\begin{array}{c}\alpha \\
(\mathrm{deg})\end{array}$ \\
\hline (35671) 1998 SN165 & 12 Aug. 02 & TNO & 38.071 & 37.284 & 0.98 \\
2001 QF298 & 12 Aug. 02 & Plutino & 42.625 & 41.740 & 0.68 \\
2002 GZ32 & 9 Mar. 03 & Centaur & 20.903 & 19.989 & 1.09 \\
(55 576) 2002 GB10 & 9 Mar. 03 & Centaur & 5.188 & 14.325 & 1.91 \\
2002 GO9 & 9 Mar. 03 & Centaur & 14.058 & 13.434 & 3.23 \\
(55 565) 2002 AW197 & 9 Mar. 03 & Cubewano & 47.260 & 46.448 & 0.69 \\
2003 AZ84 & 9 Mar. 03 & Plutino & 45.888 & 45.417 & 1.09 \\
(50 000) Quaoar & 9 Mar. 03 & Cubewano & 43.409 & 43.297 & 1.31 \\
(47 932) 2000 GN171 & 12 Apr. 03 & Plutino & 28.601 & 27.599 & 0.05 \\
\hline
\end{tabular}

of Jupiter and Neptune while the scattered disk is composed of objects with larger elliptical orbits beyond Neptune and at high inclination.

Very little is known about these objects and their study can provide information about the primordial processes that governed the evolution of the solar system and those of other disks around young stars.

After the first discovery of 15760 (1992 QB1) by Jewitt \& Luu (1992) the number of these trans-Neptunian objects grew with a veritable explosion in the last decade. More than $700 \mathrm{ob}-$ jects are now known, but distinguishing them in terms of observable properties is still in its infancy. Barucci et al. (2004) give a complete review on the physical knowledge available to date on these small icy bodies. To investigate the surface properties of these primitive objects a large program was started at ESO (in Cerro Paranal and La Silla) to observe them with visible and near-infrared photometry and spectroscopy during April 2001 to March 2003. Photometric data has allowed the study of the colour behaviour for more than 100 objects and statistical analysis of colour variations with orbital parameters (Boehnhardt et al. 2002; Peixinho et al. 2003; Peixinho et al. 2004). Spectroscopy has allowed the investigation of the surface properties and composition (Barucci et al. 2002; Lazzarin et al. 2002; Dotto et al. 2002; Doressoundiram et al. 2003).

In this paper we report new visible spectrophotometric data on 9 objects observed at the VLT-ESO (Very Large Telescope, $8 \mathrm{~m}$ telescope) with the instrument FORS1 at unit 1 Antu and unit 3 Melipal, and FORS2 at unit 4 Yepun. Details of the observing conditions and the dynamical class of the objects are reported in Table 1. A discussion of these results is presented as well as the analysis of all the visible spectral observations obtained during the large program and those existing in the literature.

\section{Observations and data reduction}

The data were obtained during three different observing runs at the Very Large Telescope: two runs in the framework of the Large Program on TNOs and Centaurs on 11 August 2002 and 8 March 2003, plus another run on 12 April 2003 (071.C-500,
PI C. de Bergh) completely devoted to the surface investigation of 2000 GN171.

During the first run, two TNO's spectra were acquired at UT3 Melipal, using FORS1 instrument; the night was clear but with a highly variable seeing $\left(0.6-1.9^{\prime \prime}\right)$; the spectra of two TNOs have been acquired. The second run we observed at UT1 Antu, again using FORS1, which had been moved from UT3 Melipal; the night was photometric with good seeing conditions $\left(<0.9^{\prime \prime}\right)$ and we obtained three spectra of Centaurs and three of TNOs. The last run, performed at UT4 Yepun with the FORS2 instrument, was totally devoted to the TNO 2000 GN171, with the aim of studying the surface composition during its rotational period, determined by Sheppard \& Jewitt (2002) to be of $8.33 \pm 0.05 \mathrm{~h}$. Unfortunately we lost the first $3 \mathrm{~h}$ of observations due to clouds, but the remainder of the night was clear with a good seeing $\left(0.6-1^{\prime \prime}\right)$. We acquired 6 spectra of this TNO covering more than half of its rotational period.

In Table 2 all the details of the spectroscopic observations are given, including date and UT time of the acquisition, the telescope and the instrument used, the exposure time and the object airmass (mean of the airmass at the beginning and at the end of observation), and finally the solar analog star used to remove the solar contribution.

All the spectroscopic observations, both with FORS1 and FORS2 instrument, were performed using a low resolution grism (150 grooves/mm) with a $1^{\prime \prime}$ wide slit, covering the 4000-9200 ^ wavelength range with a spectral resolution of about 200. The slit was oriented along the parallactic angle to minimize the effects of atmospheric differential refraction, which is also corrected by a system of two silica prism (named Longitudinal ADC) implemented both at FORS1 and FORS2 instruments.

The FORS1 detector is a TK2048EB4-1 backside thinned CCD of $2048 \times 2048$ pixel, with a pixel size of $24 \mu \mathrm{m}$, corresponding to a pixel scale of $0.2^{\prime \prime} / \mathrm{px}$; the high gain mode (1.45 $\mathrm{e}^{-} /$ADU) was used for the read out of the CCD both for spectroscopy and imaging.

The FORS2 detector is a mosaic of two MIT/LL CCD of $4096 \times 2048$ pixel each (pixel size $24 \mu \mathrm{m}$, corresponding to a pixel scale of $0.126^{\prime \prime} / \mathrm{px}$ ), used in a $2 \times 2$ binned mode; 
Table 2. Observational conditions of the TNOs and Centaurs spectroscopically investigated. For each object we report the observational date and universal time (UT of the beginning of the exposure), the VLT unit and instrument used, the total exposure time, the airmass (mean of the airmass value at the beginning and at the end of observation), and the observed solar analog stars with their airmass used to remove the solar contribution.

\begin{tabular}{l|lllllll}
\hline \hline Object & Date & UT & Telescope & Instr. & $\begin{array}{c}T_{\text {exp }} \\
\text { (min.) }\end{array}$ & Airm. & Solar An. (airm.) \\
\hline Centaurs & & & & & & & \\
\hline 2002 GZ32 & 9 Mar. 03 & $6: 16$ & UT1 (ANTU) & FORS1 & 60 & 1.24 & Landolt 98-978 (1.09) \\
(55 576) 2002 GB10 & 9 Mar. 03 & $4: 44$ & UT1 (ANTU) & FORS1 & 60 & 1.06 & Landolt 102-1081 (1.10) \\
2002 GO9 & 9 Mar. 03 & $7: 46$ & UT1 (ANTU) & FORS1 & 60 & 1.09 & Landolt 107-998 (1.13) \\
\hline TNOs & & & & & & & \\
\hline (35 671) 1998 SN165 & 12 Aug. 02 & $7: 22$ & UT3 (MELIPAL) & FORS1 & 50 & 1.11 & Landolt 115-271 (1.12) \\
2001 QF298 & 12 Aug. 02 & $5: 32$ & UT3 (MELIPAL) & FORS1 & 40 & 1.09 & Landolt 115-271 (1.12) \\
(55 565) 2002 AW197 & 9 Mar. 03 & $2: 38$ & UT1 (ANTU) & FORS1 & 60 & 1.22 & Landolt 98-978 (1.09) \\
2003 AZ84 & 9 Mar. 03 & $1: 09$ & UT1 (ANTU) & FORS1 & 60 & 1.31 & Landolt 107-998 (1.13) \\
(50 000) Quaoar & 9 Mar. 03 & $9: 31$ & UT1 (ANTU) & FORS1 & 15 & 1.03 & Landolt 107-998 (1.13) \\
(47 932) 2000 GN171 & 12 Apr. 03 & $4: 23$ & UT4 (YEPUN) & FORS2 & 30 & 1.04 & Landolt 107-998 (1.10) \\
(47 932) 2000 GN171 & 12 Apr. 03 & $5: 25$ & UT4 (YEPUN) & FORS2 & 30 & 1.07 & Landolt 107-998 (1.10) \\
(47 932) 2000 GN171 & 12 Apr. 03 & $6: 09$ & UT4 (YEPUN) & FORS2 & 30 & 1.14 & Landolt 107-998 (1.10) \\
(47 932) 2000 GN171 & 12 Apr. 03 & $7: 07$ & UT4 (YEPUN) & FORS2 & 30 & 1.33 & Landolt 107-998 (1.24) \\
(47 932) 2000 GN171 & 12 Apr. 03 & $7: 50$ & UT4 (YEPUN) & FORS2 & 30 & 1.58 & Landolt 107-998 (1.48) \\
(47932) 2000 GN171 & 12 Apr. 03 & $8: 33$ & UT4 (YEPUN) & FORS2 & 40 & 2.04 & Landolt 107-998 (1.48) \\
\hline
\end{tabular}

the low gain read out $\left(1.25 \mathrm{e}^{-} / \mathrm{ADU}\right)$ was used during imaging acquisitions while for spectroscopy observations we utilized the high gain read out $\left(0.77 \mathrm{e}^{-} / \mathrm{ADU}\right)$. During each night we also acquired bias, flat-field, calibration lamp (He-Ar) and several solar analog stars spectra at different intervals throughout the night.

For the solar analog stars two consecutive exposures were acquired, one of which with a order sorting filter (OG590) cutting the signal below $5900 \AA$ to avoid the overlapping of the second order beyond $8000 \AA$. The influence of the second order was estimated to be quite low, but to avoid possible contamination, we used a combined spectrum for each solar analog star: below $6500 \AA$ we used the star spectrum acquired without the sorter filter, beyond $7500 \AA$ the star spectrum obtained with the sorter filter and in the $6500-7500 \AA$ region the mean of the two spectra obtained with and without the filter.

All the minor bodies spectra were acquired without the sorter filter, as the influence of the second order is estimated to be lower than in the case of the bright solar analog stars. To verify this we compared the solar analog spectra acquired with and without the sorter filter, deriving a wavelength dependent function for the second order influence. Combining opportunely this function with the blue part of the objects spectra we verify that the second order influence is completely negligible below $8600 \AA$, less than $1 \%$ in the $8600-8900 \AA$ spectral range and more than $1 \%$ only for longer wavelengths. As our spectra finish around $9200 \AA$, we considered the second order influence negligible in the Centaurs and TNOs spectra.

The total exposure time indicated in Table 2 was obtained by adding two consecutive exposures (that is a total time of $60 \mathrm{~min}$ was obtained with two exposure of $30 \mathrm{~min}$ each). This observing strategy allows us to minimize the cosmic rays hitting on the frames and also to check and eventually correct the position of the object inside the slit. Only 50000 Quaoar and 2000 GN171 were observed in a single acquisition, as Quaoar was very bright and for 2000 GN171 we needed single exposures to properly investigate possible surface composition variations.

Spectra were reduced using normal data reduction procedures (see Lazzarin et al. 2003) with the software package Midas. The wavelength calibration was performed using helium, $\mathrm{HgCd}$, and argon lamp spectral lines. The reflectivity of the objects was obtained by dividing their spectra by the spectrum of the solar analog star closest in time and airmass, as reported in Table 2.

Spectra have been normalized at $5500 \AA$ and finally smoothed with a median filter technique, using a box of $39 \AA$ in the spectral direction for each point of the spectrum. Threshold was set to 0.1 , meaning that the original value was replaced by the median value when this last differed by more than $10 \%$ from the original one.

Finally, we computed the slope of the continuum using a standard least squared technique for a linear fitting to the spectrum in the wavelength range between $5000 \AA$ and $8000 \AA$.

During August 2002 and March 2003 observing runs imaging observations have been performed using the broadband Bessel $B-V-R-I$ filter of FORS1 instrument, centered respectively at $4290 \AA, 5540 \AA, 6570 \AA$ and $7680 \AA$. The photometric observations were made just before the spectroscopy. To eliminate systematic errors in the colors caused by rotational lightcurve variation, we adopted the following photometric sequence: $R-V-B-I-V$. Unfortunately $B$ filter observations were not available for the TNOs 1998 SN165 and 2001 QF298. The imaging data were reduced in a standard manner and 
Table 3. Results of the photometric observations: $V$ magnitude, $B-V, V-R$ and $V-I$ colors. We also report the date of the observations and the UT relative to the beginning of the photometric acquisition sequence, corresponding to the $R$ filter. The acquisition sequence, taken just before the spectral observations, was usually in the form $R-V-B-I-V ; B$ filter observations were not available for the TNOs 1998 SN165 and 2001 QF298. For 2002 AW197, 2002 GB10, 2002 GO9 and 2002 GZ32 the exposure time was of $60 \mathrm{~s}$ in $R$ and $V$ filters, $180 \mathrm{~s}$ in $B$ and $120 \mathrm{~s}$ in $I$; for 2003 AZ84: $90 \mathrm{~s}$ in $R$ and $V$ filters, $240 \mathrm{~s}$ in $B$ and $180 \mathrm{~s}$ in $I$; for 50000 Quaoar: $30 \mathrm{~s}$ in $R$ and $V, 90 \mathrm{~s}$ in $B$ and $30 \mathrm{~s}$ in $I$; for 1998 SN165 and 2001 QF298: $180 \mathrm{~s}$ in $R$ and $V, 240 \mathrm{~s}$ in $I$.

\begin{tabular}{l|cccccc}
\hline \hline Object & Date & UT $_{\text {start }}$ & $V$ & $B-V$ & $V-R$ & $V-I$ \\
\hline Centaurs & & & & & & \\
\hline 2002 GZ32 & 9 Mar. 03 & $5: 53$ & $20.60 \pm 0.02$ & $0.71 \pm 0.03$ & $0.50 \pm 0.03$ & $0.91 \pm 0.03$ \\
(55 576) 2002 GB10 & 9 Mar. 03 & $4: 22$ & $19.99 \pm 0.02$ & $1.12 \pm 0.03$ & $0.72 \pm 0.03$ & $1.32 \pm 0.03$ \\
2002 GO9 & 9 Mar. 03 & $7: 24$ & $20.83 \pm 0.03$ & $1.13 \pm 0.03$ & $0.76 \pm 0.03$ & $1.44 \pm 0.03$ \\
\hline TNOs & & & & & & \\
\hline (35671) 1998 SN165 & 12 Aug. 02 & $6: 50$ & $21.58 \pm 0.04$ & Not avail. & $0.44 \pm 0.05$ & $0.93 \pm 0.05$ \\
2001 QF298 & 12 Aug. 02 & $4: 50$ & $21.77 \pm 0.04$ & Not avail. & $0.43 \pm 0.05$ & $0.82 \pm 0.05$ \\
(55 565) 2002 AW197 & 9 Mar. 03 & $2: 17$ & $20.44 \pm 0.02$ & $0.90 \pm 0.03$ & $0.62 \pm 0.03$ & $1.18 \pm 0.03$ \\
2003 AZ84 & 9 Mar. 03 & $0: 37$ & $20.47 \pm 0.02$ & $0.65 \pm 0.03$ & $0.39 \pm 0.03$ & $0.75 \pm 0.03$ \\
(50 000) Quaoar & 9 Mar. 03 & $8: 54$ & $19.27 \pm 0.02$ & $0.94 \pm 0.02$ & $0.67 \pm 0.02$ & $1.28 \pm 0.02$ \\
\hline
\end{tabular}

calibrated using different photometric standard stars observed during each night (Landolt 1992); all the details about imaging acquisition and reduction are reported in Peixinho et al. (2004). The obtained $V$ magnitude and $B-V, V-R$ and $V-I$ colors are reported in Table 3.

Concerning April 2003 run, for 2000 GN171 we performed imaging observations before each spectral acquisition using the FORS2 $R$ filter (centered at $6550 \AA$ ) in order to retrieve the rotational phase of the spectral data. Exposure times were typically of $60 \mathrm{~s}$ for each $R$ image. As the night was clear but not photometric, only relative photometry between the object and some comparison field stars was possible.

\section{Results}

\subsection{GN171}

In the framework of the Large Program on physical studies of Trans-Neptunians objects 2000 GN171 was observed twice. This interest is due to the fact that for the first time the spectrum of a TNO revealed a weak feature centered around $7250 \AA$ (Lazzarin et al. 2003). Only 2000 EB173, observed during the same night of $2000 \mathrm{GN171}$, reveals two other weak features centered around 6000 and $7300 \AA$. The origin of these bands is still not well understood, but they look similar to some features found on low albedo asteroids and associated to hydrated silicates derived from aqueous alteration. De Bergh et al. (2004) analyse the possible interpretations of these peculiar bands, on the basis also of the infrared data $(1.2-2.5 \mu \mathrm{m})$ available for 2000 GN171 and 2000 EB173, concluding that aqueous alteration seems at this moment the most plausible source of the features observed.

In order to confirm the peculiar features detected on April 2001, the observations of both 2000 GN171 and 2000 EB173 were repeated on 10 May 2002 at the VLT UT3 with the FORS1 instrument. These latest visible spectra are presented in de Bergh et al. (2004): they are quite different from the April 2001 observations, in particular there is no evidence for the absorption bands previously revealed. The different spectral behaviour was interpreted to be due to surface composition heterogeneities observed during the TNO's rotation. To check this possibility, observing time independent of the Large Program was requested by C. de Bergh at the VLT telescope to investigate 2000 GN171 during a complete rotational period. As previously mentioned, the first part of the night was lost due to clouds, so we were able to cover only about the $3 / 5$ of its rotational period (the total spectral observations cover about $5 \mathrm{~h}$, while Sheppard \& Jewitt (2002) gives a rotational period of $8.33 \mathrm{~h}$ ).

The six spectra obtained are shown in Fig. 1. We label the first spectrum obtained A, the second one B and so on until the sixth, labelled F. The first striking result is that none of the six spectra shows any absorption feature (the weak absorption around $8200 \AA$ on $\mathrm{C}, \mathrm{D}$ and $\mathrm{E}$ spectra is due to an incomplete removal of $\mathrm{H}_{2} \mathrm{O}$ telluric band). So the material responsible for the weak band revealed on April 2001 must be located on the opposite side with respect to the portion of the surface we observed. From relative photometry we construct the composite lightcurve, represented in Fig. 2, with the zero phase corresponding at Julian Date 2452742.67. The photometric acquisitions were made just before the spectral ones, so the A-F letters indicated in Fig. 2 correspond to about the beginning of each spectral acquisition. The partial lightcurve obtained shows a high magnitude variation for 2000 GN171, with a maximum difference of $0.5 \mathrm{mag}$, a result consistent with the peak to peak variation of $0.61 \pm 0.03$ mag found by Sheppard $\&$ Jewitt (2002). It must be noted that the $8.33 \mathrm{~h}$ period is the best solution found by these authors for their photometric data, with a double peaked curve that explained also the asymmetry of 0.08 mag between the two upper and lower peaks of their data. However also a weaker solution with a rotational period of $4.17 \mathrm{~h}$ and a single peak lightcurve was found. The double peak solution with the longer rotational period is the most plausible as it is expected for rotational modulation caused by an aspherical shape. Moreover the fact that we had not found a 


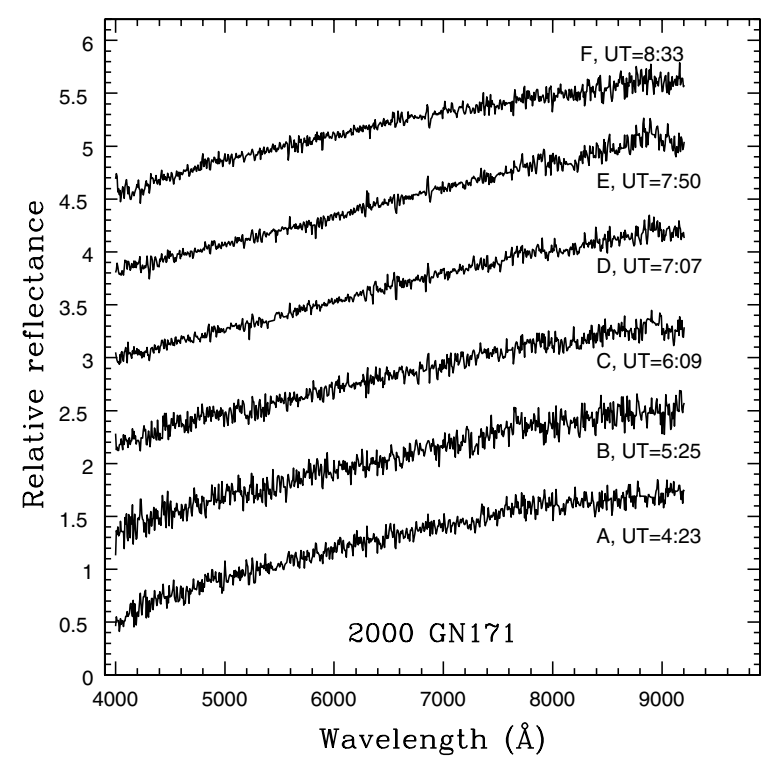

Fig. 1. Visible spectra of 2000 GN171 obtained during part of its rotational period. All spectra have been normalized at $5500 \AA$ and shifted by 0.8 for clarity. The first acquired spectrum is labelled A, the second $\mathrm{B}$ and so on till the sixth (F). The UT time at the beginning of each observation is also indicated.

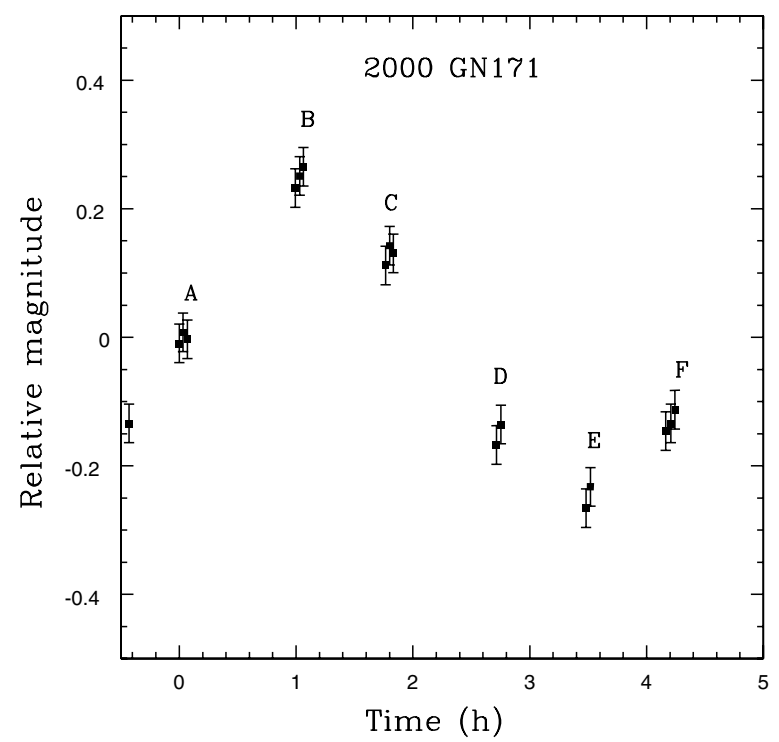

Fig. 2. Composite lightcurve of $2001 \mathrm{GN} 171$ obtained by relative photometry. The error bars contain the photon noise. The zero phase is at JD 2452742.67 . We place above the data the letters A-F indicating the corresponding spectrum (represented in Fig. 1) taken just after each photometric data set.

spectrum similar to that observed on April 2001 during about $5 \mathrm{~h}$ of observations argue against the single peak solution.

Considering that the 2 peaks in the Sheppard \& Jewitt lightcurve differs by 0.08 mag and that our data have a maximal variation of $0.5 \mathrm{mag}$, we conclude that our spectral coverage corresponds to the peak with lower amplitude variation (secondary peak) referring to Sheppard \& Jewitt lightcurve.

The spectral slope of the six 2000 GN171 spectra and of the mean spectrum resulting from their average is reported in Table 4. The first 3 spectra (see Fig. 1), taken
Table 4. Reflectance slope and relative errors (evaluated in the 5000$8000 \AA$ wavelength range) for the six obtained spectra (named A-F as explained in the text) of 2000 GN171. We put the slope value of $F$ spectrum inside square bracket as it could be affected by errors in the extinction correction process due to the low airmass value. The slope value of the spectrum obtained by the mean of the six different spectra is also given.

\begin{tabular}{lc}
\hline \hline Object & Slope $\left(\% / 10^{3} \AA\right)$ \\
\hline 2000 GN171 & \\
\hline A & $23.3 \pm 0.3$ \\
B & $23.5 \pm 0.4$ \\
C & $23.7 \pm 0.3$ \\
D & $26.1 \pm 0.3$ \\
E & $26.9 \pm 0.3$ \\
F & {$[20.9 \pm 0.3]$} \\
mean & $24.1 \pm 0.2$ \\
\hline
\end{tabular}

around the maximum of our partial lightcurve peak, are practically undistinguishable with an almost constant spectral slope around $23.5 \% / 10^{3} \AA$. The fourth and the fifth spectra show a progressive increasing in the spectral slope till to about $27 \% / 10^{3} \AA$. The last spectrum $(\mathrm{F})$, taken after the peak minimum in our lightcurve, shows a decreasing of the spectral slope $\left(21 \% / 10^{3} \AA\right)$. However this spectrum was acquired at an high airmass (around 2) and its reflectivity obtained using a solar analog with a quite different airmass (around 1.5), so the spectral slope value obtained, quite different from the previous ones, might be in part affected by a less than ideal extinction correction; for this reason this spectral slope value needs to be confirmed by independent observations, even if it seems to indicate a decreasing trend in the spectral slope near the secondary minimum of the lightcurve.

The previous visible spectral data of 2000 GN171 give a spectral slope of $20.7 \pm 0.3 \% / 10^{3} \AA$ (evaluated on the 4000-9000 ̊ spectral range) for the April 2001 observations (Lazzarin et al. 2003) and of $16.3 \pm 0.2 \% / 10^{3} \AA$ (evaluated on 5000-8000 A range) for the May 2002 ones (de Bergh et al. 2004). As the April 2001 observations were close to the determination of 2000 GN171 rotational period, we can derive quite precisely their position on the lightcurve, that is around 0.7 and 0.78 of the phase curve, just after the primary maximum. So they were effectively placed in a different region of the lightcurve in comparison to the data presented here and which do not show any evidence of the absorption bands previously detected. Concerning May 2002 observations, the current uncertainty on the rotation period of 2000 GN171 cannot allow us to precisely position them on the lightcurve, as they seem to be placed around $0.55 \pm 0.15$ of the phase curve, after the secondary minimum of the lightcurve.

The variations in the spectral slopes computed during the same observing night and in different runs, together with the detection of a peculiar absorption band on April 2001 that has not been revealed by successive observations on different lightcurve regions, seem to indicate that this Plutino is an intriguing object with an heterogeneous surface composition. 
Table 5. Reflectance slope and relative errors for all the Centaurs and TNOs observed spectroscopically in the visible range; the objects perihelion distance $(q)$ and their diameter (derived from radiometric and/or thermal and optical measurements, when available, or estimated assuming an albedo of $0.04\left({ }^{a}\right.$ Fernandez et al. 2002; ${ }^{b}$ Davies et al. 1993; ${ }^{c}$ Jewitt \& Kalas 1998; ${ }^{d}$ Margot et al. 2002; ${ }^{e}$ Thomas et al. 2000)), are also given. References (Ref.) for the spectral slopes are: 1. This work; 2. Lazzarin et al. (2003); 3. Barucci et al. (1999); 4. Binzel (1992); 5. Luu \& Jewitt (1998); 6. Luu \& Jewitt (1996); 7. Boehnhardt et al. (2001); 8. Marchi et al. (2003); 9. de Bergh et al. (2004); 10. Fitzsimmons et al. (1994); 11. Lazzaro et al. (1997); 12. Boehnhardt et al. (2004).

\begin{tabular}{|c|c|c|c|c|c|c|c|c|c|}
\hline Object & $\begin{array}{c}\text { Slope } \\
\left(\% / 10^{3} \AA\right)\end{array}$ & $\begin{array}{c}D \\
(\mathrm{~km})\end{array}$ & $\begin{array}{c}q \\
(\mathrm{AU})\end{array}$ & Ref. & Object & $\begin{array}{c}\text { Slope } \\
\left(\% / 10^{3} \AA\right)\end{array}$ & $\begin{array}{c}D \\
(\mathrm{~km})\end{array}$ & $\begin{array}{c}q \\
(\mathrm{AU})\end{array}$ & Ref. \\
\hline Centaurs & & & & & TNOs & & & & \\
\hline 2002 GB10 & $34.3 \pm 0.1$ & 121 & 15.188 & 1 & 2002 AW197 & $22.1 \pm 0.2$ & $886^{d}$ & 41.390 & 1 \\
\hline 2002 GZ32 & $8.3 \pm 0.1$ & 183 & 18.093 & 1 & 2003 AZ84 & $3.3 \pm 0.2$ & 840 & 32.724 & 1 \\
\hline 2002 GO9 & $35.8 \pm 0.1$ & 61 & 14.039 & 1 & 50000 Quaoar & $26.9 \pm 0.2$ & 1230 & 41.806 & 1 \\
\hline 2000 EC98 & $23.7 \pm 0.6$ & 64 & 15.644 & 2 & 50000 Quaoar & $28.2 \pm 0.2$ & 1230 & 41.806 & 8 \\
\hline 1999 OX3 & $52.5 \pm 0.7$ & 210 & 17.539 & 2 & 1998 SN165 & $8.8 \pm 0.4$ & 457 & 36.077 & 1 \\
\hline 2001 PT13 & $11.1 \pm 0.2$ & 90 & 8.522 & 2 & 2001 QF298 & $5.8 \pm 0.4$ & 732 & 34.761 & 1 \\
\hline 2000 QC243 & $10.1 \pm 0.2$ & 190 & 13.136 & 2 & 2000 GN171 & $24.1 \pm 0.2$ & 416 & 28.304 & 1 \\
\hline 1998 SG35 & $11.8 \pm 0.2$ & 35 & 5.806 & 2 & 2000 GN171 & $20.7 \pm 0.3$ & 416 & 28.304 & 2 \\
\hline 2001 BL41 & $16.4 \pm 0.2$ & 21 & 6.895 & 2 & 2000 GN171 & $16.2 \pm 0.2$ & 416 & 28.304 & 9 \\
\hline 2060 Chiron & $-0.5 \pm 0.2$ & $148^{a}$ & 8.436 & 3 & 2000 EB173 & $24.2 \pm 0.4$ & 732 & 28.566 & 2 \\
\hline 2060 Chiron & $-0.5 \pm 0.2$ & $148^{a}$ & 8.436 & 10 & 1999 KR16 & $55.6 \pm 0.7$ & 457 & 34.036 & 2 \\
\hline 2060 Chiron & $2.3 \pm 0.2$ & 148 & 8.436 & 11 & 1999 ТC36 & $30.6 \pm 0.2$ & 675 & 30.541 & 2 \\
\hline 2060 Chiron & $0.4 \pm 0.1$ & 148 & 8.436 & 11 & 1996 GQ21 & $34.9 \pm 0.3$ & 602 & 38.228 & 2 \\
\hline 5145 Pholus & $55.0 \pm 2.0$ & $190^{b}$ & 8.698 & 4 & 1999 DE9 & $25.5 \pm 0.2$ & 732 & 32.298 & 2 \\
\hline 7066 Nessus & $57.9 \pm 0.9$ & 75 & 11.773 & 3 & 1996 TL66 & $-1.1 \pm 0.7$ & 632 & 35.034 & 5 \\
\hline 8405 Asbolus & $17.0 \pm 0.4$ & $66^{a}$ & 6.836 & 3 & $157891993 \mathrm{SC}$ & $20.0 \pm 9.0$ & $328^{e}$ & 32.195 & 6 \\
\hline 10199 Chariklo & $7.8 \pm 0.1$ & $302^{c}$ & 13.074 & 3 & 1996 TO66 & $3.0 \pm 3.0$ & 761 & 38.019 & 7 \\
\hline 10370 Hylonome & $10.7 \pm 1.1$ & 151 & 18.856 & 3 & 1995 SM55 & $-12.0 \pm 3.0$ & 717 & 37.350 & 7 \\
\hline TNOs & & & & & 1998 HK151 & $7.0 \pm 3.0$ & 200 & 30.363 & 7 \\
\hline 28978 Ixion & $17.9 \pm 0.4$ & 1055 & 29.881 & 8 & 1997 CQ29 & $27.0 \pm 5.0$ & 316 & 39.886 & 7 \\
\hline 28978 Ixion & $22.2 \pm 0.2$ & 1055 & 29.881 & 12 & $1997 \mathrm{CS} 29$ & $27.0 \pm 3.0$ & 602 & 43.428 & 7 \\
\hline
\end{tabular}

\subsection{Centaurs}

The new spectra of the three Centaurs together with their colors indices transformed in spectral reflectance are represented in Fig. 3. They look quite different since two objects (2002 GO9 and 2002 GB10) are red, with a spectral slope value around $35 \% / 10^{3} \AA$ (Table 5), while 2002 GZ32 is gray, with a moderately small spectral gradient $\left(\sim 8 \% / 10^{3} \AA\right)$. There is a good agreement between spectral and imaging data, except for the $V-I$ color in 2002 GO9 and the $B-V$ color in 2002 GB 10.

All the three Centaur spectra were acquired during the same observing run; the gray object, 2002 GZ32, has a flat spectrum with a moderate increase in the spectral slope around $7600 \AA$; this object has an indication of a weak absorption band centered around $4300 \AA$ (wide $200 \AA$ and depth of about $3 \%$ as respect to the continuum); this feature can be seen in both the two different consecutive exposures and also using different solar analog stars, and it seems to be real. However the presence of solar features around the $4300 \AA$ together with the drop off of the detector sensitivity in this region make this identification uncertain and other observations with a higher signal to noise ratio in the blue region are needed to really confirm this band. If real, this feature would be similar to the absorption at $4300 \AA$ found on some primitive main belt asteroids and attributed to a ferric iron $\mathrm{Fe}^{3+}$ spin forbidden absorption present in minerals derived from the aqueous alteration process such as iron sulfate jarosite (Hunt \& Ashley 1979; Townsend 1987; Vilas et al. 1993a). As discussed in de Bergh et al. (2004) the aqueous

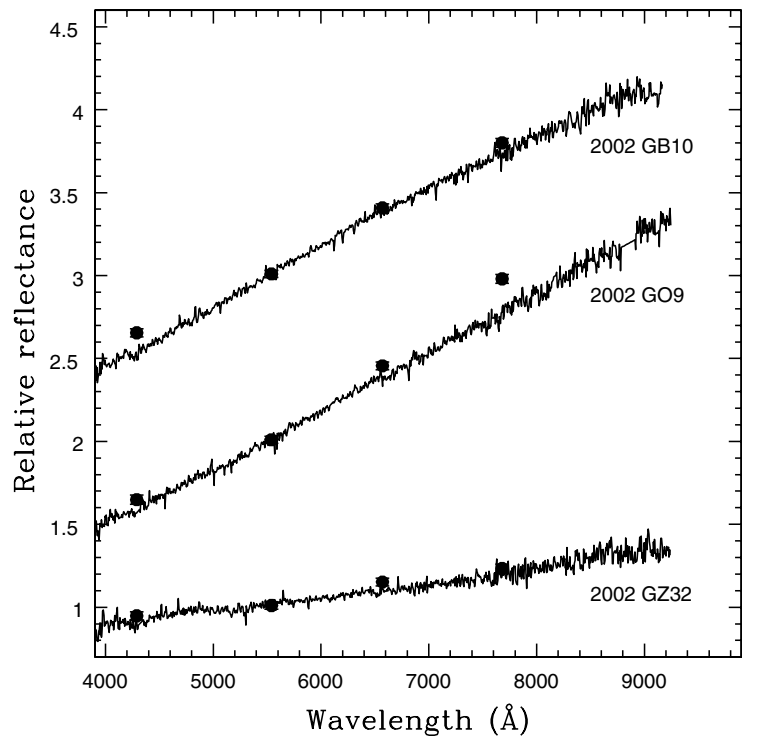

Fig. 3. Visible spectra of the three Centaurs observed. Spectra have been normalized at $5500 \AA$ and shifted of 0.7 for clarity. The colors indices transformed in spectral reflectance are also shown on each spectrum.

alteration process might also have taken place also at these large heliocentric distances with an adequate thermal process, even if alternative explanations may be found. For example, Cochran \& Vilas (1997) found a band at $4300 \AA$ on asteroid 4 Vesta attributed to a spin forbidden $\mathrm{Fe}^{2+}$ crystal field 


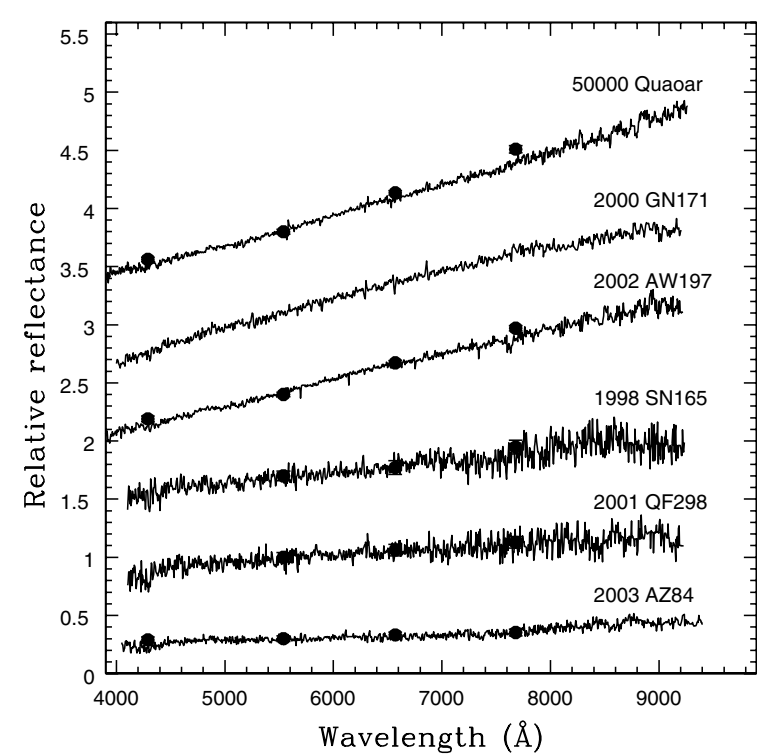

Fig. 4. Visible spectra of the six TNOs. Spectra have been normalized at $5500 \AA$ and shifted of 0.7 for clarity. The colors indices transformed in spectral reflectance are also shown on each spectrum. For 2000 GN171 we represented here the mean spectrum of the six different exposures available.

transition in pyroxene, that nevertheless is unlikely to be present in large amounts on Centaurs and TNOs surfaces, as the typical 1 and $2 \mu \mathrm{m}$ pyroxene bands have not yet been found in their spectra.

The other two Centaurs are reddish with a steep increasing reflectance; no absorption features have been identified. The weak absorption around $4300 \AA$ on 2002 GB10 (of smaller width in comparison to that seen on 2002 GZ32) is very likely due to an incomplete removal of the solar absorption features due to a combination of Fe I (4308 $\AA$ ), Cr I (4290 ̊) and CH $(4292,4302,4311 \AA$ ) lines (an example of the solar absorption features around $4300 \AA$ is reported in Vilas et al. 1993a, Fig. 4).

No other visible spectroscopic data are available in the literature for these Centaurs.

\subsection{Trans-Neptunians}

The new spectra of the six TNOs together with their color indices transformed in spectral reflectance are represented in Fig. 4 (for 2000 GN171 we used a mean of the six spectra available from April 2003 observations), and their slopes are reported in Table 5.

The objects investigated look quite different, comprising bluish to gray and red members: three members are moderately red (2002 AW197, 2000 GN171 and 50000 Quaoar) with a spectral slope around $20-27 \% / 10^{3} \AA$ and three are gray (2002 AZ84, 2001 QF198 and 1998 SN165) with a spectral gradient smaller than $9 \% / 10^{3} \AA$ (Table 5). Color indices transformed in reflectance show a very good agreement with the spectral data.

The three red objects do not show any evidence of absorption features and have a steeply increasing slope; among the

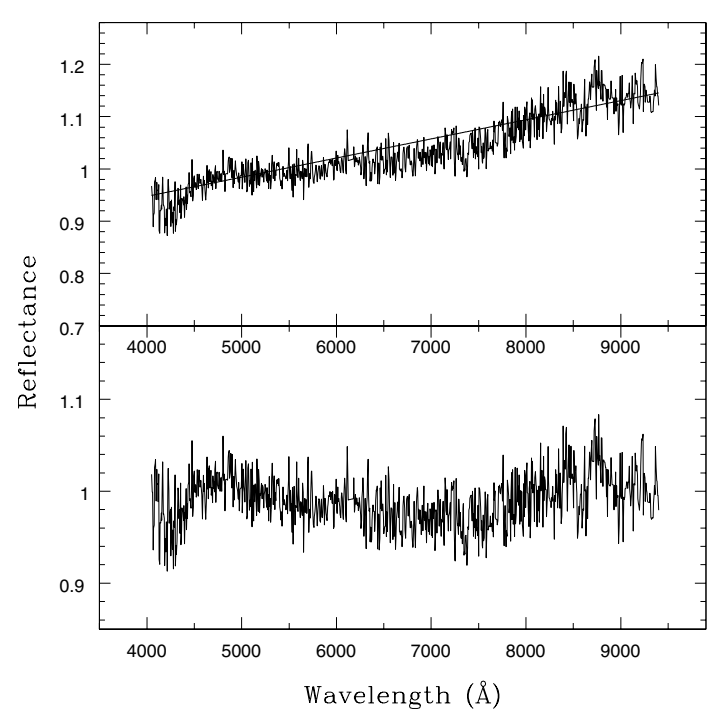

Fig. 5. Top: visible spectrum of 2003 AZ84 with superimposed the linear continuum computed with a linear least squares fit to the smoothed spectral data; bottom: spectrum of 2003 AZ84 with the continuum removed.

bluish TNOs, some weak absorption features are present on 2003 AZ84, that is one of the gray objects in the TNOs population. In fact, looking carefully at the 2003 AZ84 spectrum (Fig. 5), it is possible to identify a broad absorption band centered around $7000 \AA$, very similar to that observed on primitive main belt asteroids affected by aqueous alteration. This band is present in both the two separate spectra acquired, when they are averaged to improve the signal to noise ratio and also when using different solar analog stars. To confirm the existence of this band we computed a linear continuum by a linear least squares fit to the smoothed spectral data points; we then divided the spectrum by the continuum, thus removing a sloping background (Fig. 5) following the same procedure described by Vilas et al. (1993b) and Fornasier et al. (1999) for the analysis of aqueous alteration bands on primitive main belt asteroids. The band is weak, with a depth of about $3 \%$ with respect to the continuum and more than $3000 \AA$ wide. In comparison with the $7000 \AA$ band seen on main belt asteroids, this one seems to begin around $5000 \AA$, that is at shorter wavelength with respect to the typical band lower edge, usually around 5300-5500 $\AA$. The upper edge is not easy to identify due to an incomplete removal of the telluric bands beyond $8000 \AA$ but seems to be placed around $8500 \AA$.

On low albedo main belt asteroids and meteorites this band has been attributed to an $\mathrm{Fe}^{2+} \rightarrow \mathrm{Fe}^{3+}$ charge transfer in iron oxides in phillosilicates (Vilas \& Gaffey 1989; Vilas et al. 1993a).

The spectrum of 2003 AZ84 also seems to show a weak absorption centered around $4300 \AA$ and about $200 \AA$ wide. This band is similar to that possibly present in the spectrum of 2002 GZ32, and, as previously explained, future observations with an higher signal to noise ratio in the blue spectral range are needed to confirm its reality. Other weak features present at $8500 \AA, 8800 \AA$ and $9200 \AA$ are due to an incomplete removal of telluric bands and/or of solar features. 
The other two gray objects, 1998 SN165 and 2001 QF298, are noisier with a poorer removal of telluric absorption bands beyond $8000 \AA$. A very weak feature in the $0.7-0.8 \mu \mathrm{m}$ region seems to be present in the spectrum of 1998 SN165, however this band is very faint and inside the peak to peak scatter of the data. This very weak absorption is similar to those found by Lazzarin et al. (2003) on 2000 GN171 and 2000 EB173 and tentatively attributed to aqueous alteration; observations with a higher signal to noise ratio are needed to confirm it.

Regarding 2001 QF298, there is a faint absorption around $4300 \AA$, similar to that seen on 2002 GB10. This is very likely due to incomplete removal of the solar features.

\section{Discussion}

The absorption band centered around $7000 \AA$ found on 2003 AZ84, and those centered around $4300 \AA$ possibly present on the spectra of 2003 AZ84 and 2002 GZ32, seem to be very similar and compatible with the absorption features found on low albedo main belt asteroids and due to aqueous alteration.

The aqueous alteration process requires liquid water (stable for about 10 million years) to occur, so a source of water, probably in the form of ice, and a thermal process, for example the decay of radionuclides such as the ${ }^{26} \mathrm{Al}$ and/or electrical induction heating due to a strong early solar wind during the T-Tauri phase of the Sun (Jones et al. 1990) are needed to make the process effective. Water ice is expected to be abundant in the outer solar system and in fact it has been found on the surface of many giant planets satellites and also on some Centaurs and TNOs (Luu et al. 2000; Brown et al. 1999; Cruikshank et al. 1998; Barucci et al. 2002; Licandro et al. 2003; Dotto et al. 2003). The main problem in making aqueous alteration effective on outer solar system minor bodies is related to the thermal process: induction heating seems to be less and less efficient at increasing heliocentric distances, and no evidence of water signatures has been found on C and D-type asteroids located beyond 3.5 AU. Alternative source of heating on Centaurs and TNOs might come from radiogenic heating or collisional impacts, but we do not know if these processes may be sufficient for aqueous alteration of minerals. Also the possibility that hydrated minerals might have been formed just in the early solar system and not only successively by the action of the aqueous alteration process on anhydrous materials cannot be excluded (see for example Grossman \& Larimer 1974; Robert 2001). A complete discussion about the possible action of the aqueous alteration process on TNOs and Centaurs is reported in de Bergh et al. (2004).

On main belt bodies the $4300 \AA$ band is often accompanied by the $7000 \AA$ feature (Vilas et al. 1993a); the possible presence of the $4300 \AA$ band on 2002 GZ32 without that of the $7000 \AA$ band may be explained by the absence of iron bearing silicates on their surfaces, as proposed for other main belt asteroids (see Cochran \& Vilas 1997).

In Table 5 we report all the visible spectral slopes available from this work and in the literature for Centaurs and TNOs. The sample contains 15 Centaurs and 19 TNOs, whose spectral investigation in the visible range comes prevalently from the Large Program, with 9 Centaur and 11 TNO spectra. Regarding

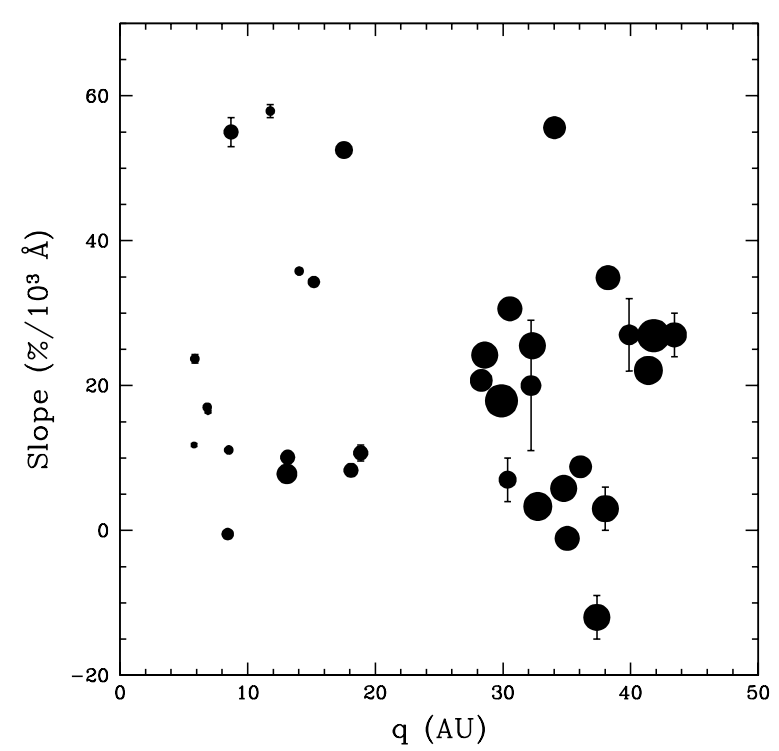

Fig. 6. Plot of the spectral slope versus the perihelion distance for all the centaurs and TNOs whose visible spectrum is available from the Large Program and in literature. The size of the points is proportional to the estimated diameter of the bodies. The smaller points represent the objects with estimated diameters less than $50 \mathrm{~km}$, and their size progressively increases in steps of $50 \mathrm{~km}$ for the Centaurs and $100 \mathrm{~km}$ for the TNOs according to the values given in Table 5. The biggest points correspond to TNOs with an estimated diameter larger than $900 \mathrm{~km}$.

the new spectra presented here, only 479322000 GN171 and 50000 Quaoar have been previously observed by other authors. For 2000 GN171 the spectral slopes reported in this work, Lazzarin et al. (2003) and de Bergh et al. (2004) are different but due to variations of the surface composition of this TNO, as previously described. Its near infrared spectrum is quite flat, except in the $\mathrm{H}$ band where a broad absorption appears (de Bergh et al. 2004). For 50000 Quaoar our spectral slope is in good agreement with that reported by Marchi et al. (2003). This large TNOs has been recently observed in the infrared range (1.4-2.4 $\mu \mathrm{m})$, showing the presence of some absorption features such as crystalline $\mathrm{H}_{2} \mathrm{O}$ ice and $\mathrm{CH}_{4}$ as reported by Brown \& Trujillo (2004). Infrared spectral observations obtained in the framework of the Large Program are also available for (55565) $2002 \mathrm{AW} 197$, (55576) 2002 GB10 and 2002 GO9 and the results are presented in Doressoundiram et al. (2004).

In Fig. 6 we represent the spectral slope versus the perihelion distance of all the TNOs and Centaurs whose visible spectral slope is known. The symbol size is proportional to object diameter. First of all the large variety in the spectral behaviour of both the Centaur and TNO populations is confirmed, since the spectral slope spans a wide range of colors, from gray to very red.

The spectral slope and the object diameter seem not to be correlated; moreover, an observation bias strongly affects the TNOs population, where only the largest objects can be discovered and observed.

Similar results were found also by McBride et al. (2003) analysing a sample of 29 Kuiper Belt objects with previously 


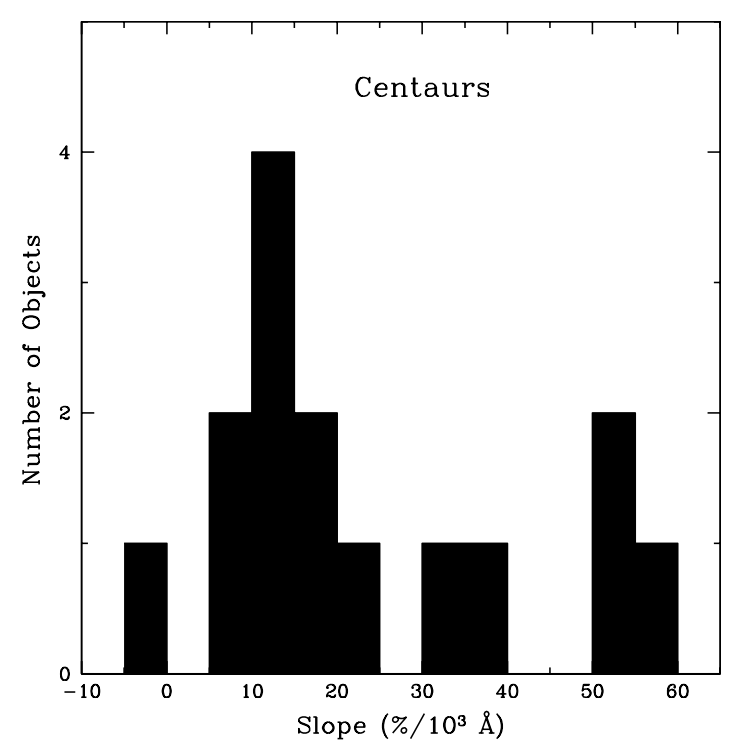

Fig. 7. Distribution of the Centaurs as a function of the spectral slope. The sample includes 15 objects observed via visible spectroscopy from the ESO Large Program and in the literature.

published optical colors and new obtained simultaneously $V-$ $J$ color. In fact they confirm the variety of slopes of the reflected sunlight in the optical-infrared spectral range and they do not find any evidence of a color-size relationship. They find a reasonable correlation between the optical band relative reflectance spectra and the visual-infrared relative reflectance spectra, concluding that probably a single reddening agent worked on the Kuiper Belt objects' surfaces. The red slopes of TNOs shown in McBride et al. (2003) and Barucci et al. (2001) have been modeled using a combination of organic tholins and amorphous carbon by Cruikshank \& Dalle Ore (2004). Their model spectra can admit, but do not require the addition of space weathered, igneous rock-forming minerals to fit the TNOs spectral behaviours.

McBride et al. (2003) also report the trend that there seems to be a predominance of red material on the surface of objects having perihelia beyond $40 \mathrm{AU}$, as previously found by Tegler \& Romanishin (2000). Considering our data, the only 3 objects (Quaoar, 2002 AW197 and 1997 CS29) having perihelia beyond $40 \mathrm{AU}$ are red or moderately red, with quite similar spectral slopes varying from about 22 to $28 \% / 10^{3} \AA$. Although our data seem to confirm the trend previously described by McBride et al. (2003) and Tegler \& Romanishin (2000), we believe that our sample is too limited and that more spectrophotometric observations are needed to clearly identify a possible relationship with color/spectral slopes for objects with perihelium $>40$ AU.

For a better analysis of the Centaurs and TNOs spectral slope distribution we represent in Figs. 7 and 8 the number of objects as a function of spectral slope for the two distinct groups. Figure 7 tell us that most part of the Centaurs (the $66 \%$ ) are bluish-gray or moderately red objects, with spectral slope lower than $25 \% / 10^{3} \AA ; 20 \%$ of them are very red, with a spectral slope $>50 \% / 10^{3} \AA$, while a lower percentage, $13 \%$, corresponds to red objects with spectral slope

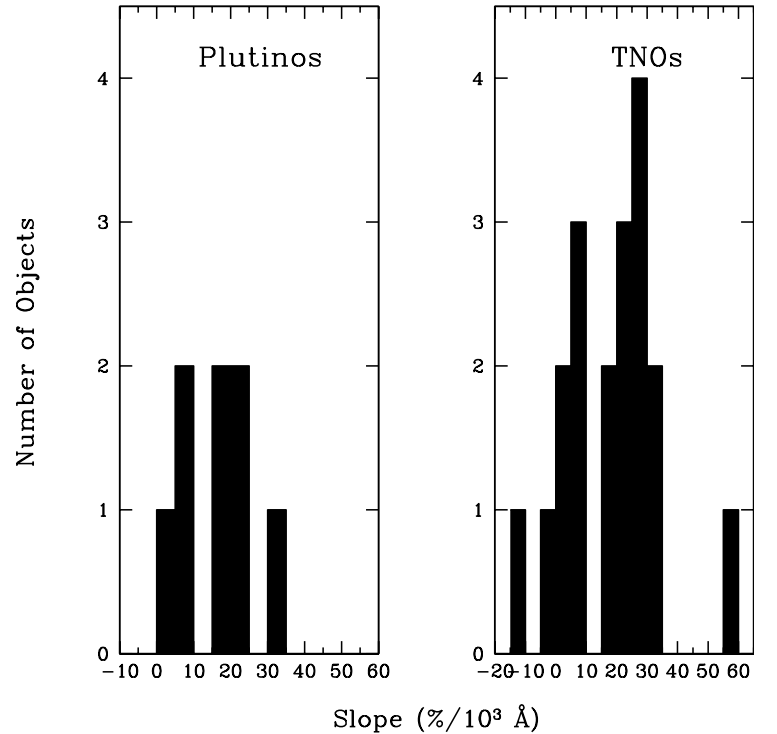

Fig. 8. Distribution of the Plutinos (left) and of the TNOs (right) as a function of the spectral slope. The sample comprises 19 objects observed via visible spectroscopy from the ESO Large Program and in the literature.

between 30 and $40 \% / 10^{3} \AA$, in the transition region from moderately to very red objects.

If we compare the distribution for Centaurs with that of the TNOs (Fig. 8), it is evident that there is a lack of very red objects in the TNOs sample investigated, as only one object (the $5 \%$ of the sample) has a spectral slope greater than $40 \% / 10^{3} \AA$. Most of the TNOs $(58 \%)$ are red or moderately red objects, with spectral slope between 15 and $35 \% / 10^{3} \AA$, while $42 \%$ of the sample comprises bluish to gray objects, with spectral slope lower than $10 \% / 10^{3} \AA$.

If we look only at the Plutinos in our TNOs sample, the same trend is confirmed, as most of them $(62.5 \%)$ are red or moderately red objects, while $37.5 \%$ are bluish to gray objects. No very red Plutinos have been spectroscopically observed to date.

This spectral variety confirms the results obtained by several authors from their photometric investigation on a larger sample of TNOs and Centaurs (Luu \& Jewitt 1996; Barucci et al. 2000; Doressoundiram et al. 2001, 2002; Jewitt \& Luu 2001; Delsanti et al. 2001; Tegler \& Romanishin 2000; Boehnhardt et al. 2002; McBride et al. 2003). Tegler \& Romanishin (1998), from their TNOs and Centaurs color analysis conclude that two distinct populations exist, one having neutral to slightly red colors, and one with the reddest objects known in the Solar System. This bimodality was not supported by several authors using the increasing data set available (Tegler \& Romanishin 2000; Boehnhardt et al. 2001; Doressoundiram et al. 2002; Hainaut \& Delsanti 2002), and actually it has been confirmed only for the Centaurs population, as shown by Peixinho et al. (2003).

From our smaller data set on spectroscopic observations nothing can be derived regarding grouping in the Centaur and TNO populations. We may only underline that there is a gap in the distribution of both Centaurs and TNOs for a spectral 
slope between 40 and $50 \% / 10^{3} \AA$, but we cannot state if it is real or due to the small spectroscopy data set available. The gap between the two distinct Centaur groups in the $V-R$ versus $B-V$ plot in Peixinho et al. (2003) corresponds to a spectral slope of about $20 \% / 10^{3} \AA$ (assuming $B-V \sim 0.95$ and $V-R \sim 0.58)$, a value that is in the middle between the spectral reflectance of Asbolus $\left(17 \% / 10^{3} \AA\right)$ and that of 2000 EC98 (23.7\%/10 ${ }^{3} \AA$, Table 5).

The diversity in the spectral behaviour and so presumably in the surface composition can be explained by different degrees of surface alteration due to the balance between aging and rejuvenating processes, such as space weathering, collisions and cometary activity (Delsanti et al. 2004). The dominance of blue-gray and moderately red objects in the Centaurs may be caused by a present or past cometary activity (Chiron is a typical example) which has rejuvenated part of the population.

\section{Conclusions}

In this work we have presented new visible spectrophotometric data on six TNOs and three Centaurs. We have analysed these new data together with all the spectroscopic data available from the ESO Large Program on TNOs and Centaurs and in literature. The main results obtained may be summarized as follows:

- The spectra of the Centaurs and TNOs investigated show a large variety of spectral behaviour. This confirms the heterogeneity in the surface composition of the Centaur and TNO population derived by the photometric investigation on a larger sample of bodies.

- Absorption features: we have revealed a weak absorption band on the gray TNOs 2003 AZ84, centered around $7000 \AA$ and extending from $5000 \AA$ to $8500 \AA$, with a depth of about the $3 \%$ as respect to the continuum. This feature is very similar to the typical broad absorption band observed in the visible spectra of many low albedo main belt asteroids and produced by the action of aqueous alteration. In addition, 2003 AZ84 and the Centaurs 2002 GZ32 may have a weak absorption around $4300 \AA$, that may be also associated with aqueous alteration products such as iron sulfate jarosite. Future observations with a higher signal to noise ratio in the blue spectral region are needed to confirm the possible presence of the $4300 \AA$ band.

- From previous data from the Large Program, very peculiar visible absorption bands on 2000 GN171 and 2000 EB173 have been identified and aqueous alteration claimed as being possibly responsible for these features (Lazzarin et al. 2003; de Bergh et al. 2004). Now the detection of the $7000 \AA$ band on 2003 AZ84 with a very similar location and spectral width to the typical broad visible band due to the action of aqueous alteration process on main belt asteroids seems to confirm that the aqueous alteration process might have taken place at large heliocentric distances such as those of TNOs.

How this process might be effective so far away from the Sun is not yet understood: possible source of heating on TNOs might be radiogenic heating or impacts, but it cannot be excluded that hydrated minerals could have been formed directly in the early solar nebula.

- (47 932) 2000 GN171: after the very first detection of a peculiar absorption band on April 2001, we have repeated the observations of this TNO in order to confirm this absorption. The spectrum obtained on May 2002 did not have any absorption feature, indicating that this object has probably an heterogeneous surface composition. On April 2003 we observed 2000 GN171 during one night, obtaining 6 spectra distributed over more than half of its rotational period. No absorption features have been found and we conclude that we observed a different surface region in comparison to the April 2001 data. The slight differences in the spectral slope of the 8 spectra obtained together with the detection of a peculiar absorption band on April 2001 seem to indicate that this Plutino is an intriguing object with an heterogeneous surface composition.

- Considering all the visible spectral data available (15 objects), the Centaur population is dominated (66\%) by bluish to gray or moderately red objects but comprises also very red objects $(20 \%)$, with spectral slopes greater than $50 \% / 10^{3} \AA$. This large variety and the dominance of bluishmoderately red objects compared to the TNOs probably reflects strong resurfacing processes from cometary activity or collisions.

- The 19 TNOs which have been spectroscopically investigated in the visible region are predominantly red or moderately red objects (the $58 \%$ ), but there is a lack of very red objects in comparison with the Centaurs, as only one object has a spectral slope greater than $40 \% / 10^{3} \AA$. In particular no very-red objects have been spectroscopically investigated until now in the Plutinos population.

- No relationship between the size of the objects and the spectral gradient has been found.

\section{References}

Barucci, M. A., Lazzarin, M., \& Tozzi, G. P. 1999, AJ, 117, 1929

Barucci, M. A., Romon, J., Doressoundiram, A., \& Tholen, D. J. 2000, AJ, 496

Barucci, M. A., Boenhardt, H., Dotto, E., et al. 2002, A\&A, 392, 335

Barucci, M. A., Doressoundiram, A., \& Cruikshank, D. P. 2004, in Comets II, ed. M. Festou et al. (University of Arizona Press), in press

Binzel, R. P. 1992, Icarus, 99, 238

Boehnhardt, H., Tozzi, G. P., Birkle, K., et al. 2001, A\&A, 378, 653

Boehnhardt, H., Delsanti, A., Barucci, M. A., et al. 2002, A\&A, 395, 297

Boehnhardt, H., Bagnulo, S., Muinonen, K., et al. 2004, A\&A, 415, L21

Brown, R. H., Cruikshank, D. P., \& Pendleton, Y. 1999, ApJ, 519, L101

Brown, M. E., \& Trujillo, C. A. 2004, ApJ, submitted

Cochran, A. L., \& Vilas, F. 1997, Icarus, 127, 121

Cruikshank, D. P., Roush, T. L., Bartholomew, M. J., et al. 1998, Icarus, 135, 389

Davies, J. K., Sykes, M. V., \& Cruikshank, D. P. 1993, Icarus, 102, 166 
de Bergh, C., Boehnhardt, H., Barucci, M. A., et al. 2004, A\&A, 416, 791

Delsanti, A. C., Boehnhardt, H., Barrera, L., et al. 2001, A\&A, 380, 347

Delsanti, A. C., Hainaut, O., Jourdeuil, E., et al. 2004, A\&A, 417, 1145

Doressoundiram, A., Barucci, M. A., Romon, J., \& Veillet, C. 2001, Icarus, 154, 277

Doressoundiram, A., Peixinho, N., de Bergh, C., et al. 2002, AJ, 124, 2279

Doressoundiram, A., Tozzi, G. P., Barucci, M. A., et al. 2003, AJ, 125, 2721

Doressoundiram, A., Tozzi, G. P., Boehnhardt, H., et al. 2004, in preparation

Dotto, E., Barucci, M. A., Boehnhardt, H., et al. 2003, Icarus, 162, 408

Fernandez, Y. R., Jewitt, D. C., \& Sheppard, S. S. 2002, AJ, 123, 1050

Fitzsimmons, A., Dahlgren, M., Lagerkvist, C. I., Magnusson, P., \& Williams, I. P. 1994, A\&A, 282, 634

Fornasier, S., Lazzarin, M., Barbieri, C., \& Barucci, M. A. 1999, A\&AS, 135, 65

Grossman, L., \& Larimer, J. W. 1974, Rev. Geophys. Space Phys., 12, 71

Hainaut, O. R., \& Delsanti, A. C. 2000, A\&A, 389, 641

Hunt, G. R., \& Ashley, R. P. 1979, Econ. Geol., 74, 1613

Jewitt, D., \& Kalas, P. 1998, ApJ, 499, L103

Jewitt, D., \& Luu, J. X. 2001, AJ, 122, 2099

Jones, T. D., Lebofsky, L., Lewis, J., \& Marley, M. 1990, Icarus, 88, 172

Landolt, A. U. 1992, AJ, 104, 340
Lazzarin, M., Barucci, M. A., Boehnhardt, H., et al. 2003, AJ, 125, 1554

Lazzaro, D., Florczak, M. A., Angeli, C. A., et al. 1997, Plan. Space Sci., 45, 1607

Licandro, J., Campins, H., de Leon Cruz, J., Gil-Hutton, R., \& Lara-Lopez, L. M. 2003, Am. Astron. Soc., DPS Meeting, 35, 39.11

Luu, J. X., \& Jewitt, D. C. 1996, AJ, 111, 499

Luu, J. X., \& Jewitt, D. C. 1998, ApJ, 494, L117

Luu, J. X., Jewitt, D. C., \& Trujillo, C. 2000, AJ, 531, L151

Marchi, S., Lazzarin, M., Magrin, S., \& Barbieri, C. 2003, A\&A, 408, L17

Margot, J. L., Trujillo, C., Brown, M. E., \& Bertoldi, F. 2002, BAAS, 34,871

McBride, N., Green, S. F., Davies, J. K., et al. 2003, Icarus, 161, 501

Peixinho, N., Doressoundiram, A., Delsanti, A., et al. 2003, A\&A, 410, L29

Peixinho, N., Boehnhardt, H., Belskaya, I., et al. 2004, Icarus, in press Robert, F. 2001, Science, 293, 1056

Sheppard, S. S., \& Jewitt, D. C. 2002, AJ, 124, 1757

Tegler, S. C., \& Romanishin, W. 1998, Nature, 392, 49

Tegler, S. C., \& Romanishin, W. 2000, Nature, 6807, 979

Thomas, N., Eggers, S., Ip, W. H., et al. 2000, ApJ, 534, 446

Townsend, T. E. 1987, J. Geophys. Res., 92, 1441

Vilas, F., \& Gaffey, M. J. 1989, Science, 246, 790

Vilas, F., Hatch, E. C., Larson, S. M., Sawyer, S. R., \& Gaffey, M. J. 1993a, Icarus, 102, 225

Vilas, F., Larson, S. M., Hatch, E. C., \& Jarvis, K. S. 1993b, Icarus, 105,67 